\title{
Geociências
}

\section{Fonte e grau da contaminação por hidrocarbonetos policíclicos aromáticos (HPAs) de baixa massa molecular em sedimentos da baía de Todos os Santos, Bahia}

\author{
Joil José Celino \\ Dr. em Geologia Regional. Núcleo de Estudos Ambientais. Instituto de Geociências \\ Universidade Federal da Bahia (UFBA), Rua Barão de Geremoabo, s/n, Sala 305 A1, Federação \\ CEP 40170-290, Salvador -BA - Brasil.E-mail: joil@ufba.br \\ Antônio Fernando de S. Queiroz \\ Dr. em Geoquímica de Manguezais. Núcleo de Estudos Ambientais, Instituto de Geociências \\ Universidade Federal da Bahia (UFBA), Rua Barão de Geremoabo, s/n, Sala 305 A1, Federação \\ CEP 40170-290, Salvador - BA - Brasil.E-mail: queiroz@ufba.br
}

\section{Resumo}

A baía de Todos os Santos (BTS), localizada no Recôncavo Baiano, é a maior baía navegável do Brasil. Com aproximadamente $450 \mathrm{~km}$ de extensão, sua orla é recoberta por extenso manguezal e sedimentos recentes. Esse estudo foi desenvolvido para avaliar a possibilidade do impacto ambiental crônico induzido pelos 50 anos de exposição do local à indústria do petróleo. Hidrocarbonetos policíclicos aromáticos (HPAs) provenientes de fonte pirolítica foram identificados e quantificados em uma concentração total variando entre 1 - 408.629 ng. g $^{-1}$. As mais altas concentrações de HPAs foram encontradas nos portos e nas áreas de transporte. Os índices moleculares Phe/An e An/(An+Phe) foram usados para identificar a fonte de contaminação por HPAs nas estações de amostragem estudadas. Todavia as concentrações de HPAs pirolíticos foram comparáveis com aquelas de zonas contaminadas (áreas da refinaria e de extração). No geral, os sítios de amostragem na baía de Todos os Santos foram contaminados, devido ao transporte de petróleo em escala individual ao longo da baía e, também, pelo resíduo de óleos a partir de embarcações motorizadas.

Palavras-chave: hidrocarbonetos, pirolíticos, sedimentos, contaminação, baía de Todos os Santos.

\begin{abstract}
Todos os Santos Bay, located in Recôncavo Bahiano, is the largest navigable bay of Brazil. Its seafront has about $450 \mathrm{~km}$ and is covered by extensive mangroves and recent sediments. This study was undertaken to evaluate the possibility of chronic environmental impact induced by 50 years of exposure to the local petroleum industry. Polycyclic Aromatic Hydrocarbons (PAHs) from pyrolitic sources were identified and quantified in the total concentration range 1 - $408.629 \mathrm{ng} \cdot \mathrm{g}^{-1}$. The highest contents of PAHs were found in the harbors and transportation areas. Phe/An and $A n /(A n+P h e)$ molecular indices were used to identify the PAH contamination source in the studied sampling stations. It was found that the pyrolitics PAHs concentrations were comparable with those of contaminated zones (refinery and extraction areas). In general, the Todos os Santos Bay sampling sites were contaminated, due to the petroleum trade on an individual scale along the Bay, and also waste oils from motorized boats.
\end{abstract}

Keywords: hydrocarbons, pyrolitics, sediments, contamination, Todos os Santos Bay. 
Fonte e grau da contaminação por hidrocarbonetos policíclicos aromáticos (HPAs)...

\section{Introdução}

A báia de Todos os Santos tem sido palco de ações pioneiras na exploração do petróleo em território brasileiro. Em suas proximidades estão instaladas, desde a década de 1950, diversas atividades ligadas à indústria petrolífera (refinaria, porto, campo de produção em mar) (Figura 1 ). A região norte da baía de Todos os Santos (BTS) sofreu, na segunda metade do século XX, inúmeros acidentes ambientais envolvendo derrames de óleo.

Com relação à geomorfologia, a baía de Todos os Santos pode ser considerada como uma baía de maré (uma categoria especial de estuário formada por processos tectônicos de larga escala). A morfologia é, tectonicamente, controlada pelas falhas geológicas associadas à Bacia Sedimentar do Recôncavo. O sistema em graben da bacia promoveu a existência de diversos altos topográficos, além das numerosas ilhas dentro da baía (Lessa et al., 2000). Na região norte estão localizadas as ilhas dos Frades, Maria Guarda, Bom Jesus, Paty, das Fontes, das Vacas, Bimbarra, Cajaíba e Madre de Deus.

Suas características são claramente marinhas, pois o volume de água doce, oriunda dos diversos cursos fluviais que nela deságuam, é duas ordens de grandeza inferior ao aporte de água salgada que entra pela abertura da baía (Leão \& Dominguez, 2000). Trata-se de uma baía fortemente influenciada pelas massas oceânicas, com salinidade variando entre 28 e 36\%. Alguns fatores morfológicos (valores médios) contribuem para o baixo tempo de residência da água no seu interior: profundidade de 6,9 metros, volume de $6,39 \times 109 \mathrm{~m}^{3}$ e correntes com velocidade de $41,0 \mathrm{~cm} . \mathrm{s}^{-1}$. (Orge et al., 2000).

A granulometria dos sedimentos superficiais de fundo da baía de Todos os Santos varia desde argila a areia muito grossa (Lessa et al., 2000), com distribuição espacial diferenciada. Os sedimentos argilosos predominam na porção norte da baía, enquanto que, ao sul, verifica-se que as areias médias e grossas são mais expressivas. Sedimentos car- bonáticos e bioclastos ocorrem em diversas áreas, tendo sido alvo da exploração para fabricação de cimento. Franjas estreitas, mais ou menos contínuas, de recifes de corais bordejam as ilhas da baía de Todos os Santos (Leão \& Dominguez, 2000).

Os hidrocarbonetos são compostos químicos apolares (hidrófobos), o que limita sua solubilidade na água do mar, favorecendo a tendência de associação a partículas sólidas. Os hidrocarbonetos poliaromáticos (HPA) são produtos primários de processos de combustão incompleta a diferentes temperaturas e compreendem moléculas com 2 a 6 anéis aromáticos. Com base no peso molecular, os HPA podem ser divididos em dois grupos: aqueles com baixos pesos moleculares - objetos desse estudo - que possuem dois ou três anéis aromáticos, e os de altos pesos moleculares, compostos por quatro a seis ou sete anéis aromáticos.

Os HPA's de baixo peso molecular têm um significado de toxicidade aguda. Em função dessa toxidade, o conhecimento sobre suas fontes, comportamento e distribuição, no meio ambiente, é um requisito essencial para um melhor controle das atividades humanas responsá- veis pela contaminação e poluições ambientais, podendo influenciar a tomada de decisão quanto à limpeza e re-mediação dos locais considerados contaminados.

\section{Métodos experimentais}

\subsection{Amostragem}

Baseando-se em estudos anteriores (Tavares, 1997; Peso-Aguiar et al., 2000; Orge et al., 2000), foi estabelecido inicialmente, um levantamento geoquímico orientativo em oito (8) estações. Após a aquisição dos dados analíticos referentes a essa campanha, o número de estações das amostras foi ampliado. A escolha obedeceu aos critérios de: tipo de atividade petrolífera instalada (ativa ou inativa), estado de conservação do ecossistema e facilidade de acesso (terra e mar).

Tal levantamento consistiu na coleta sistemática de sedimentos superficiais (5,0 a 20,0 cm) do substrato do manguezal, em região de intermaré, em locais selecionados como mostrado na Figura 1 , entre os períodos de outubro de 2000 a fevereiro de 2001 (Silva, 2002) e de no-

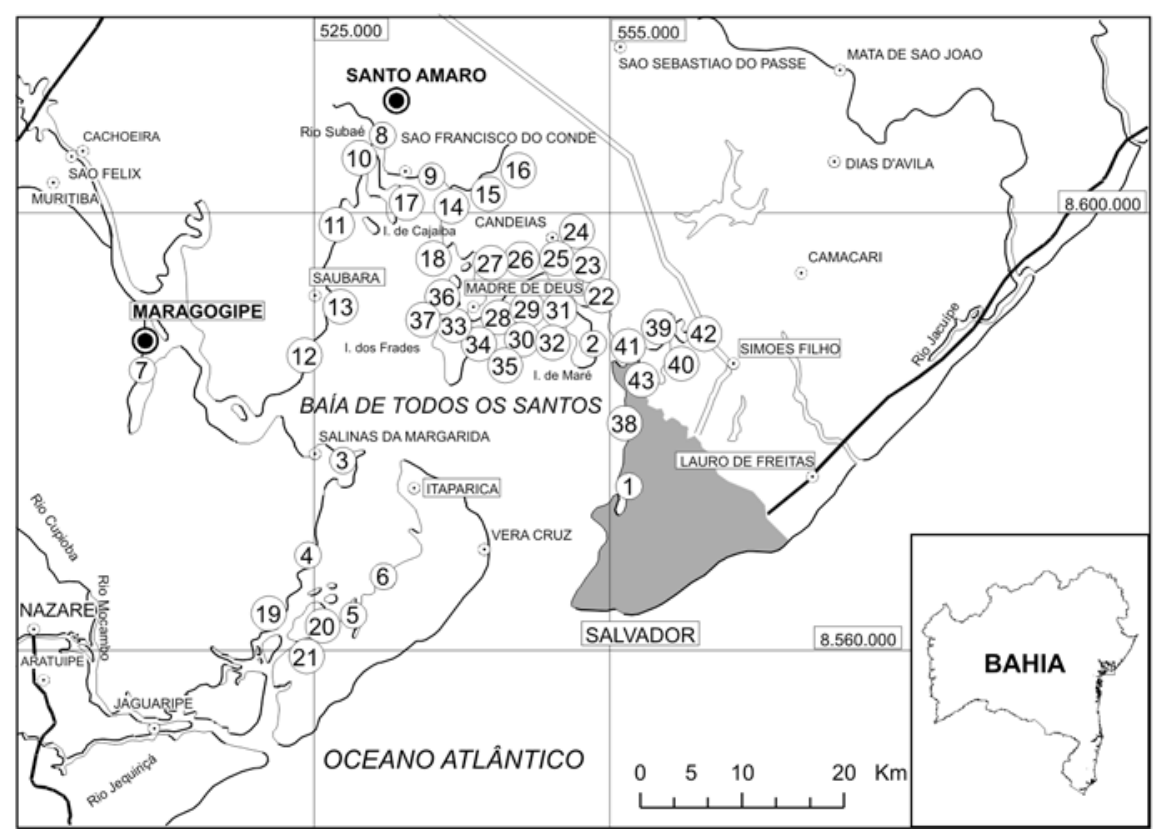

Figura 1 - Locação dos sítios de amostragem dos sedimentos na BTS. 
vembro de 2001 e setembro de 2002 (Veiga, 2003). Utilizou-se uma colher metálica lavada na água do manguezal recolhendo amostras compostas dos sedimentos superficiais até $20 \mathrm{~cm}$ de profundidade e massa de aproximadamente 500 gramas, acondicionadas em vasilhames de vidro com tampas metálicas e, imediatamente, resfriadas a temperaturas em torno de $0^{\circ} \mathrm{C}$.

\subsection{Preparação das amostras}

Uma massa de $10 \mathrm{~g}$ das amostras, sem tratamento prévio, foi fortalecida com uma solução de hidrocarbonetos policíclicos aromáticos deuterados a concentrações definidas e extraídos com diclorometano ultrapuro em soxhlet por 16 horas. O extrato foi, então, colocado em um concentrador de célula fechada do tipo Kuderna Danish a um volume de $1 \mathrm{ml}$.

\subsection{Métodos analíticos}

A determinação qualitativa e quantitativa dos HPA's obedeceu à metodologia proposta por USEPA 8279. As amostras foram analisadas por cromatografia gasosa acoplada à espectrometria de massas (CG/EM). O concentrado foi diretamente injetado, sem divisão de fluxo, $1 \mu$ do extrato em uma coluna de fase estacionária DB-5 acoplada a um espectrômetro de massas HP-MSD 5973. O espectrômetro operou em monitoramento seletivo de íons a menos de 3 ciclos por segundo. A programação de CG é descrita a seguir: injeção sem divisão de fluxo, com o injetor a $290^{\circ} \mathrm{C}$; temperatura inicial da coluna $50^{\circ} \mathrm{C}$, isoterma por $2 \mathrm{mi}-$ nutos, taxa de aquecimento de $10 \mathrm{C} / \mathrm{min}$ até a temperatura final de $310^{\circ} \mathrm{C}$, isoterma de 15 minutos.

\section{Resultados e discussões}

As concentrações totais médias dos hidrocarbonetos policíclicos aromáticos (HPA's) de baixa massa molecular expressas em ng/g estão listadas na Tabela 1 e ilustradas na Figura 2. Para o cálculo da média da concentração de HPA total, na área portuária, não foi considerada a estação de Caípe, por se tratar de uma localidade com concentração anômala, muito superior aos teores determinados nas demais estações estudadas. Quanto às médias de concentrações de HPA total de baixa massa molecular, a área de produção destaca-se como aquela de maiores valores (152,9 ng/g), seguida da área portuária (70,2 ng/g), da área de refino $(53,4 \mathrm{ng} / \mathrm{g})$ e da área de referência (2,8 ng/g). Quando se consideram os valores de concentração absolutos, verifica-se que a área portuária apresenta as maiores concentrações absolutas dos compostos HPA, variando entre 0,9 ng/g em Aratu e 408629,1ng/g em Caípe. A área de produção ocupa o segundo lugar, com concentrações variando de 13,7ng/g, em Jiribatuba, e 457,5 ng/g, em São Brás. As áreas de referência exibem os menores valores de concentração de HPA (10,2 ng/g).

Considerando-se os limites estabelecidos pela National Oceanic and Atmospheric Administration (NOAA) (Tabela 1), tanto a área de produção quanto a área portuária apresentaram valores médios de concentração maiores que os admitidos como ERM (limite médio = effective range médium), para os seguintes compostos individuais: acenafteno, fluoreno, fenantreno, antraceno, resultados esses devidos aos elevados teores de HPA detectados nas localidades de Caípe e Suape.

Considerando-se os padrões de qualidade da Environmental Canadá (1998 apud Tam et al., 2001) (Tabela 1), a área de produção apresentou concentrações superiores às estabelecidas como PQT (padrão de qualidade temporária) para os seguintes compostos: acenafteno, fluoreno, fenantreno, antraceno. Das localidades investigadas, aquelas responsáveis por esses valores anômalos são: a Ilha de Cajaíba, D. João e Jiribatuba.

As áreas de refino e de referência apresentaram padrões de qualidade compatíveis à ausência de riscos para a saúde humana, levando-se em conta as referências disponíveis na Tabela 1.
Os baixos valores de concentração de HPA apresentados pelas áreas de referência relacionam-se ao incipiente grau de urbanização da região e à ausência de instalações industriais.

Uma análise comparativa entre os principais períodos de amostragem revela que:

- As áreas de referência apresentam concentrações de HPA muito mais baixas que as demais localidades listadas, excetuando-se a área de Jiribatuba.

- Nas áreas de produção e refino, ocorreu atenuação das concentrações de HPA.

- A área portuária exibiu um agravamento do grau de comprometimento ambiental.

Guardadas as devidas precauções quanto às diferenças climáticas e dinâmicas costeiras de cada localidade, fatores que interferem diretamente na velocidade de degradação dos compostos orgânicos em ambientes marinhos e, conseqüentemente, na concentração dos mesmos em sedimentos, os resultados analíticos obtidos para a região estudada com as demais regiões globais são relativamente comparáveis.

Considerando-se as regiões costeiras e estuarinas do mundo que foram alvo de estudo (Veiga, 2003), observa-se que os valores de concentração de HPA são muito heterogêneos, variando entre 0,2 - $102.471 \mathrm{ng} / \mathrm{g}$, sendo que em zonas portuárias esses valores estão compreendidos no intervalo de 0 - $10.000 .000 \mathrm{ng} / \mathrm{g}$. Os sedimentos de zonas de manguezal da região norte da baía de Todos os Santos apresentam concentrações de HPA totais inferiores aos valores máximos citados na literatura, embora também se observe uma grande diferença entre as concentrações extremas (valores de máximo e mínimo) para cada localidade.

Considerando-se as zonas de manguezal com atividades portuárias de outras partes do mundo (Veiga, 2003), a baía de Todos os Santos apresenta um cenário de poluição mais acentuada, merecendo um estudo mais detalhado para que sejam identificados os vetores dessa poluição local. 
Fonte e grau da contaminação por hidrocarbonetos policíclicos aromáticos (HPAs)...

Tabela 1 - Descrição dos sítios de amostragem e das concentrações de Hidrocarbonetos Policíclicos Aromáticos (HPA's) de baixa massa molecular nos sedimentos coletados na BTS, expressos em ng/g.

\begin{tabular}{|c|c|c|c|c|c|c|c|c|c|c|c|}
\hline & Localidade & Amostra & Naftaleno & Acenaftileno & Acenafteno & Fluoreno & Antraceno(An) & Fenantreno(Fe) & HPAtotal(2-3 anéis) & Fe/An & $\mathrm{An} /(\mathrm{An}+\mathrm{Fe})$ \\
\hline \multirow{7}{*}{ 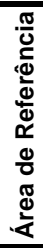 } & Cabrito & 1 & 1,70 & n.d. & n.d. & n.d. & n.d. & n.d. & 1,70 & n.c. & n.c \\
\hline & Ilha de Maré & 2 & 1,60 & n.d. & n.d. & n.d. & n.d. & n.d. & 1,60 & n.c. & n.c \\
\hline & Sal.da Marg. & 3 & 0,40 & n.d. & 0,10 & 0,10 & 0,40 & n.d. & 1,00 & n.c. & n.c \\
\hline & Mutá & 4 & 1,30 & n.d. & n.d. & n.d. & n.d. & n.d. & 1,30 & n.c. & n.c \\
\hline & Jiribatuba & 5 & 1,70 & n.d. & n.d. & n.d. & n.d. & n.d. & 1,70 & n.c. & n.c \\
\hline & Baiacu & 6 & 1,00 & 0,30 & n.d. & n.d. & 0,40 & 0,10 & 1,80 & 0,25 & 0,80 \\
\hline & Maragojipe & $7^{*}$ & 6,37 & n.d. & n.d. & n.d. & n.d. & 3,83 & 10,20 & n.c. & n.c \\
\hline \multirow{14}{*}{ 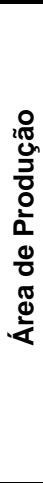 } & Subaé & $8^{*}$ & 9,49 & n.d & n.d & n.d & 2,22 & 3,65 & 15,36 & 1,64 & 0,38 \\
\hline & São F. Conde & 9 & 17,60 & 41,30 & 45,80 & 10,40 & n.d. & 56,20 & 171,30 & n.c. & n.c \\
\hline & São Brás & 10 & 10,10 & 14,00 & 16,00 & 23,50 & 43,40 & 350,50 & 457,50 & 8,08 & 0,11 \\
\hline & Acupe & 11 & 15,90 & 64,10 & 96,50 & 16,40 & 7,50 & 99,00 & 299,40 & 13,20 & 0,07 \\
\hline & Cabuçu & 12 & 21,20 & 33,80 & 48,30 & 9,50 & n.d. & 16,40 & 129,20 & n.c. & n.c \\
\hline & Saubara & 13 & 26,60 & 44,90 & 54,40 & 11,50 & 4,00 & 78,30 & 219,70 & 19,58 & 0,05 \\
\hline & Dom João & 14 & 11,00 & 0,50 & n.d. & 2,00 & 12,00 & 69,90 & 95,40 & 5,83 & 0,15 \\
\hline & Dom João & $15^{*}$ & 27,35 & 18,97 & n.d & n.d & n.d & n.d & 46,32 & n.c. & n.c \\
\hline & Dom João & $16^{*}$ & 11,24 & n.d & n.d & n.d & 1,02 & 6,28 & 18,54 & 6,16 & 0,14 \\
\hline & Ilha de Cajaíba & $17^{*}$ & 7,94 & n.d & 22,20 & 28,87 & 97,37 & 258,71 & 415,09 & 2,66 & 0,27 \\
\hline & Itha das Fontes & $18^{*}$ & 12,36 & n.d & 6,40 & 8,38 & 26,08 & 81,42 & 134,64 & 3,12 & 0,24 \\
\hline & Cações & 19 & 3,00 & 0,10 & 0,10 & 1,70 & 30,00 & 9,90 & 44,80 & 0,33 & 0,75 \\
\hline & Jiribatuba & $20^{\star * *}$ & n.d. & 0,86 & n.d. & n.d. & 4,27 & 8,57 & 13,70 & 2,01 & 0,33 \\
\hline & Jiribatuba & $21^{\star *}$ & 13,46 & 6,70 & 1,92 & 2,85 & 23,59 & 30,54 & 79,06 & 1,29 & 0,44 \\
\hline \multirow{6}{*}{ 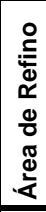 } & Coqu.Grande & $22^{*}$ & 9,88 & n.d. & n.d. & n.d. & 1,22 & 4,67 & 15,77 & 3,83 & 0,21 \\
\hline & Coqu.Grande & $23^{*}$ & 3,60 & n.d. & 1,62 & 1,08 & 3,10 & 9,79 & 19,19 & 3,16 & 0,24 \\
\hline & Coqu.Grande & $24 * *$ & n.d. & n.d. & n.d. & n.d. & 2,93 & 3,69 & 6,62 & 1,26 & 0,44 \\
\hline & Coqu.Grande & $25^{*}$ & 9,59 & 20,45 & 4,69 & n.d. & n.d. & n.d. & 34,73 & n.c. & n.c \\
\hline & Coqu.Grande & 26 & 9,00 & 15,50 & n.d. & 1,00 & 18,90 & 66,30 & 110,70 & 3,51 & 0,22 \\
\hline & Rio Mataripe & 27 & 4,50 & 0,50 & 13,00 & 15,00 & 89,40 & 11,00 & 133,40 & 0,12 & 0,89 \\
\hline \multirow{16}{*}{ 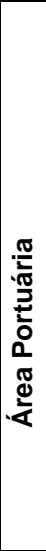 } & Madre Deus & $28^{*}$ & n.d. & n.d. & n.d. & n.d. & n.d. & 3,52 & 3,52 & n.c. & n.c \\
\hline & Caípe & 29 & 24,40 & 37,40 & 57,40 & 8,50 & n.d. & 27,90 & 155,60 & n.c. & n.c \\
\hline & Caípe & $30 *$ & 8654,35 & 312,54 & 37289,57 & 43779,98 & 91499,25 & 227093,41 & 408629,10 & 2,48 & 0,29 \\
\hline & Caípe & $31^{*}$ & n.d. & n.d. & n.d. & n.d. & n.d. & n.d. & n.c. & n.c. & n.c \\
\hline & Caípe & $32^{*}$ & n.d. & n.d. & n.d. & n.d. & n.d. & n.d. & n.c. & n.c. & n.c \\
\hline & Suape & 33 & 2,80 & 1,00 & n.d. & 16,50 & 35,40 & 158,80 & 214,50 & 4,49 & 0,18 \\
\hline & Suape & $34^{* *}$ & n.d. & n.d. & n.d. & n.d. & 2,52 & 3,79 & 6,31 & 1,50 & 0,40 \\
\hline & Suape & $35^{\star *}$ & n.d. & n.d. & 24,17 & 24,96 & 81,67 & 161,65 & 292,45 & 1,98 & 0,34 \\
\hline & Madre Deus & $36^{\star *}$ & n.d. & 0,85 & 0,66 & 0,82 & 3,18 & 4,99 & 10,50 & 1,57 & 0,39 \\
\hline & Madre Deus & $37^{\star *}$ & n.d & n.d & n.d & n.d & n.d & n.d & n.c. & n.c. & n.c \\
\hline & São T. Paripe & 38 & 0,40 & 1,00 & n.d. & n.d. & n.d. & n.d. & 1,40 & n.c. & n.c \\
\hline & Baía Aratu & 39 & 9,80 & 4,00 & 4,50 & 7,50 & 9,00 & 88,60 & 123,40 & 9,84 & 0,09 \\
\hline & Baía Aratu & 40 & 2,40 & 2,00 & 0,10 & 13,60 & 40,30 & 124,40 & 182,80 & 3,09 & 0,24 \\
\hline & Baía Aratu & 41 & 0,50 & 0,50 & n.d. & n.d. & n.d. & 3,50 & 4,50 & n.c. & n.c \\
\hline & Baía Aratu & 42 & 0,40 & 0,50 & n.d. & n.d. & n.d. & n.d. & 0,90 & n.c. & n.c \\
\hline & Baía Aratu & 43 & 18,70 & 56,60 & 57,10 & 29,80 & n.d. & 16,40 & 178,60 & n.c. & n.c \\
\hline \multirow{4}{*}{ 㣌 } & NOAA & ERL & 160,00 & 44,00 & 16,00 & 19,00 & 853,00 & 240,00 & 1332,00 & 0,28 & 0,78 \\
\hline & (Tam et al. 2001) & ERM & 2100,00 & 640,00 & 500,00 & 540,00 & 1100,00 & 1500,00 & 6380,00 & 1,36 & 0,42 \\
\hline & Environment & PQT & 34,60 & 5,87 & 6,71 & 21,20 & 46,90 & 86,70 & 201,98 & 1,85 & 0,35 \\
\hline & Canadá (1998) & NEP & 391,00 & 128,00 & 88,90 & 144,00 & 245,00 & 544,00 & 1540,90 & 2,22 & 0,31 \\
\hline
\end{tabular}

VRT $=$ Valores de Referência para Toxicidade.

NOAA - ERL = variação efetiva inferior.

$\mathrm{ERM}=$ variação efetiva média.

Environment Canadá (1998) - PQT = Padrões de qualidade temporários.

NEP = níveis de efeitos prováveis.

n.d. = não detectado.

n.c. $=$ não calculado.

limite de detecção $=0,05 \mathrm{ng} / \mathrm{g}$.

Período de amostragem = de out. 2000 a fev. 2001 (Silva, 2002).

nov. 2001* e set. 2002** (Veiga, 2003). 
Esses resultados reforçam a necessidade da implementação de uma política de monitoramento das regiões costeiras e estuarinas, com vistas a estabelecer curvas de isoteores de poluentes e a melhorar a compreensão dos seus padrões de dispersão.

\subsection{Co-variabilidade entre as concentrações de HPA's}

Os HPA's de baixa massa molecular cujas concentrações são susceptíveis de co-variação no ambiente foram identificados nesse estudo com base no valor do fator de correlação, vide Tabela 2 . Uma baixa correlação sugeriria menor quantidade de fontes de contaminação para determinada área ou a interferência de muitas fontes de HPA's, o que não foi o caso.

\subsection{Níveis de poluição nos sedimentos}

Com relação à concentração de HPA total de baixa massa molecular (Figura 2), comparada aos valores de referência de toxicidade (Environment Canadá, 1998, apud Tam et al., 2001), tanto na área de produção, quanto na área portuária, observam-se valores acima do PQT (padrões de qualidade temporários) e apenas a área portuária apresentou uma localidade com concentração média maior que a admitida pelas agências ambientais citadas na Tabela 1 (NOAA), com pico em Caípe. Essa anomalia observada na concentração de HPA em Caípe deve ser estudada detalhadamente porque, embora diversos acidentes ambientais tenham ocorrido na região em apreço, contribuindo para o estoque de HPA, esse resultado é muito maior que o obtido por Silva (2002), podendo refletir alguma heterogeneidade local que condicionou a persistência desses compostos.

\subsection{Identificação das fontes de poluição}

As razões diagnósticas baseadas em concentrações dos compostos poli-

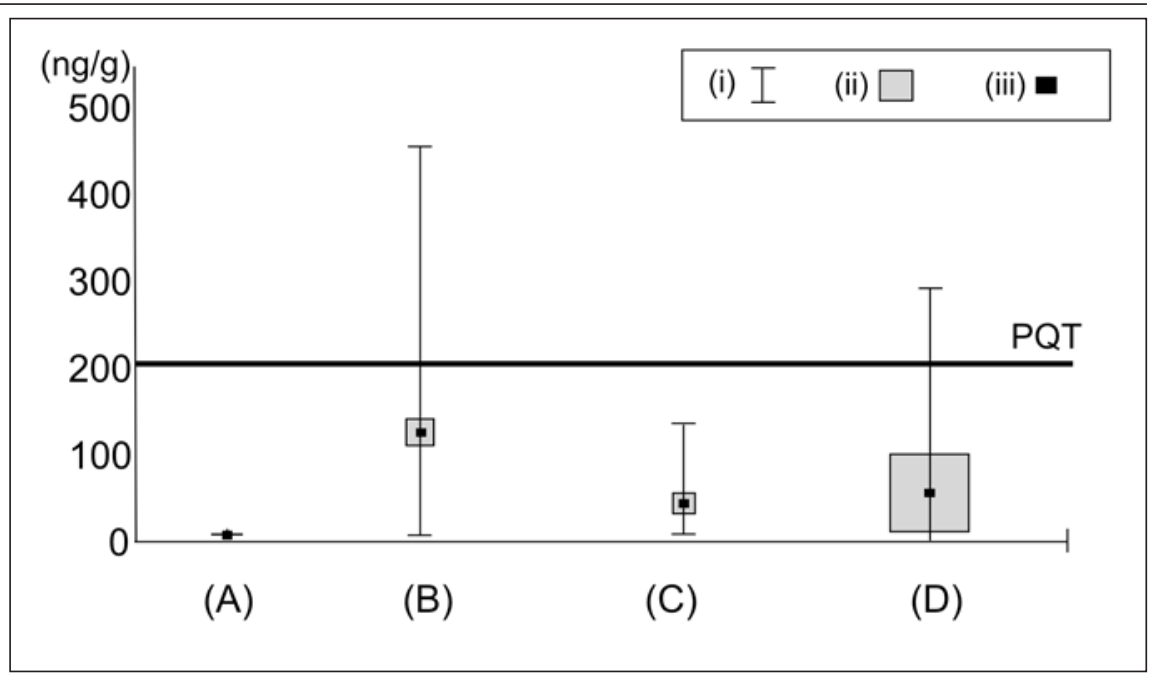

Figura 2 - Gráfico "box-plot" dos níveis de HPA's totais (ng/g), de baixa massa molecular, nos diferentes sítios de amostragem na BTS: (A) área de referência, (B) área de produção, (C) área de refino e (D) área portuária. Onde: (i) Média + Desvio-padrão; (ii) Média + erro-padrão e (iii) Média. PQT (Padrões de Qualidade Temporários) representa valor de referência para toxicidade segundo NOAA (Tam et al., 2001).

Tabela 2 - Fator de correlação entre as concentrações de Hidrocarbonetos Policíclicos Aromáticos (HPA's) de baixa massa molecular nos sedimentos coletados na BTS. Para $p<0,050$.

\begin{tabular}{ccccccc}
\hline HPA's & Naftaleno & Acenaftileno & Acenafteno & Fluoreno & Antraceno & Fenantreno \\
\hline Naftaleno & 1,00 & 0,98 & 1,00 & 1,00 & 1,00 & 1,00 \\
Acenaftileno & & 1,00 & 0,98 & 0,97 & 0,97 & 0,97 \\
Acenafteno & & & 1,00 & 1,00 & 1,00 & 1,00 \\
Fluoreno & & & & 1,00 & 1,00 & 1,00 \\
Antraceno & & & & & 1,00 & 1,00 \\
Fenantreno & & & & & & 1,00 \\
\hline
\end{tabular}

cíclicos aromáticos (Figura 3) indicaram a existência de fontes de hidrocarbonetos relacionadas a produtos da pirólise do petróleo propriamente dito em todas as áreas estudadas, com concentrações mais altas ao norte da baía, em torno das atividades da Petrobras. Ainda com relação às fontes antropogênicas de hidrocarbonetos, as mesmas também podem estar relacionadas com recreação marinha (incluindo barcos, lanchas e jet skis), em especial em áreas com forte atrativo turístico (nos entornos das ilhas e praias urbanas da região).

\section{Considerações finais}

A baía de Todos os Santos, a exemplo da baía de Guanabara, tem vivido constantemente na iminência de aciden- tes envolvendo derrames de óleo e derivados, com conseqüências graves para o meio ambiente e para a população que vive no seu entorno. Quanto aos hidrocarbonetos policíclicos aromáticos (HPAs) de baixa massa molecular, apenas a área portuária apresentou valores médios de concentração maiores que os admitidos como ERM (limite médio) pela NOAA para os seguintes compostos: acenafteno, fluoreno, fenantreno e antraceno. A área de produção apresentou concentrações superiores aos estabelecidos como PQT (padrão de qualidade temporária) para fenantreno e antraceno. As áreas de refino e controle apresentaram padrões de qualidade compatíveis à ausência de riscos para a saúde humana, no que se refere aos HPAs de baixa massa molecular. Os resultados apresentados mostraram que as zonas 
de manguezal da região norte da baía, na área de influência do complexo petrolífero instalado, têm registrado a presença de petróleo e derivados nos seus sedimentos superficiais, mesmo em áreas consideradas remotas (a exemplo de Maragogipe).

\section{Agradecimentos}

Esse trabalho é um reflexo do apoio financeiro concedido pela FINEP/RECUPETRO através do convênio de $\mathrm{n}^{\circ}$.640002700, pela Agência Nacional de Petróleo (ANP) e pelo financiamento das análises laboratoriais pela Fundação Universidade Norte Fluminense (FENORTE).

\section{Referências bibliográficas}

BUDZINSKI, H., JONES, I., BELLOCQ, J., PIÉRARD, C., GARRIGUES, P. Evaluation of sediment contamination by polycyclic aromatic hydrocarbons in the Gironde estuary. Marine Chemistry. 58, p. 85-97, 1997.

LEÃO, Z.M.A.N., DOMINGUEZ, J.M.L. Tropical coast of Brazil. Marine Pollution Bulletin. 41, p.112-122, 2000.

LESSA, G.C., LIMA, G.M., CARVALHO, J.B., OLIVEIRA, V. Oceanografia física e geologia da BTS em http:// www.cpgg.ufba.br/ glessa/bts acessado em janeiro de 2000.

ORGE, M.D.R., PORSCHÉ, I.J., COSTA, M.C., LIMA, J.S., SOARES, S.E.D., JUSTINO, R. Assessment of oil refinery waste on Rhizophora mangle L. seedling growth in mangrove of Todos os Santos Bay, Bahia, Brazil - Aquatic Ecosystem Health and Management. 3, p.471-477, 2000.

PESO-AGUIAR, M.C., SMITH, D.H., ASSIS, R.C.F., SANTA-ISABEL, L.M., PEIXINHO, S., GOUVEIA, E.P., ALMEIDA, T.C.A., ANDRADE, W.S., CARQUEIJA, C.R.G., KELMO, F., CARROZZO, G., RODRIGUES, C.V., CARVALHO, G.C., JESUS, A.C.S. Effects of petroleum and its derivatives in benthic communities at Baía de Todos os Santos, Bahia, Brazil - Aquatic Ecosystem Health and Management. 3, p. 459-470, 2000.

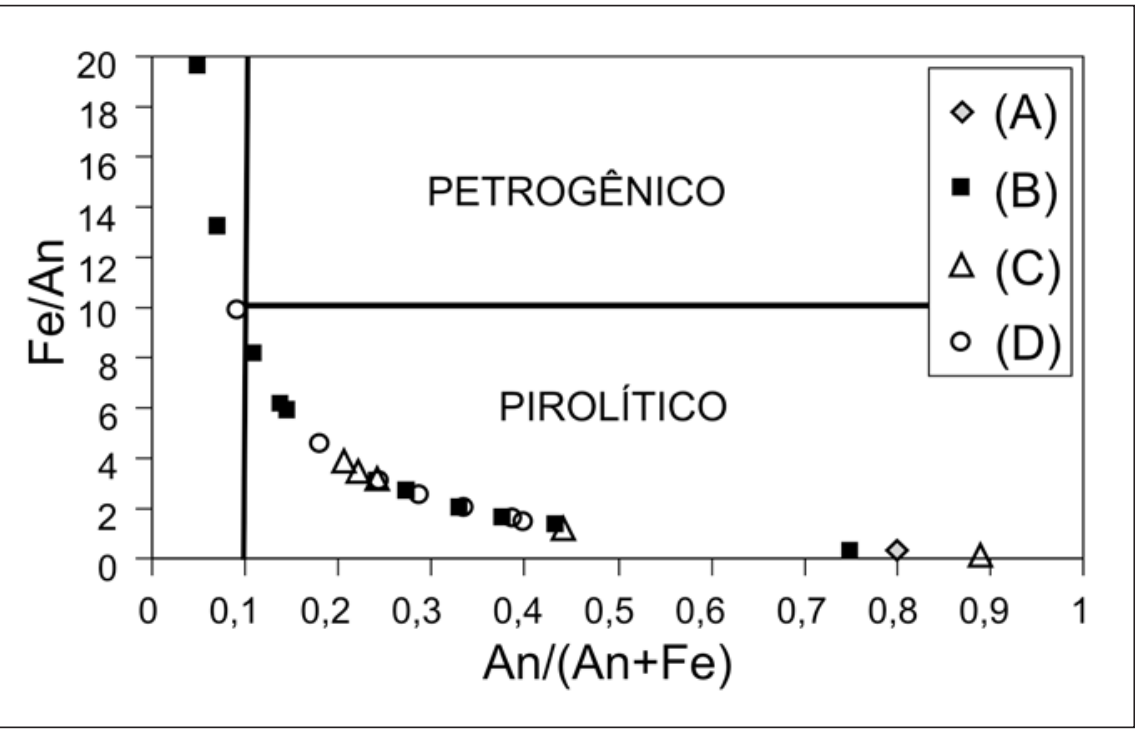

Figura 3 - Gráfico da razão Fe/An versus a razão An/(An+Fe) dos níveis de HPA's nos diferentes sítios de amostragem na BTS: (A) área de referência, (B) área de produção, (C) área de refino e (D) área portuária, com os campos das fontes segundo Yunker et al. (2002) e Budzinski et al. (1997).
SILVA, S.M.T. da. Avaliação da contaminação por HPAs individuais $e$ totais em sedimentos de mesolitoral na baía de Todos os Santos. Salvador: IQ / UFBA, 2002. 109p. (Dissertação de mestrado em Química Analítica).

TAVARES, T.M. Contaminação química no ambiente marinho. In: Baía de Todos os Santos: diagnóstico sócio-ambiental e subsídios para a gestão. Salvador: Germen / Universidade Federal da Bahia. NIMA, 1997. p. 151-164.

TAM, N.F.Y., KE, L., XANG, X.H., WONG, Y.S. Contamination of PAH in surface sediments of mangroves swamps. Environmental Pollution. 114, p. 255263, 2001.
VEIGA, I.G. Avaliação da origem dos hidrocarbonetos em sedimentos superficiais de manguezais da região norte da baía de Todos os Santos, Bahia. Macaé: LENEP/UENF, 2003. 205p. (Dissertação de mestrado em Engenharia e Exploração de Petróleo).

YUNKER, M.B., MACDONALD, R.W, VINGARZAN, R., MITCHELL, R.H., GOYETTE, D., SYLVESTRE, S. Polycyclic aromatic hydrocarbons in the Fraser River basin: a critical appraisal of $\mathrm{PAH}$ ratios as indicators of PAH sources and composition. Organic Geochemistry. 33, p. 489-515, 2002.

Artigo recebido em 05/05/2006 e aprovado em 28/08/2006.

\section{$\star * * * * *$}

\section{REM - Revista Escola de Minas 70 anos divulgando CIÊNCIA.}

\author{
$* \star * \star * *$
}

www.rem.com.br 